\title{
A parametric study on the PD pulses activity within micro-cavities
}

\author{
Alireza A. Ganjovi ${ }^{1}$
}

Received: 29 April 2015/Accepted: 17 November 2015/Published online: 7 December 2015

(C) The Author(s) 2015. This article is published with open access at Springerlink.com

\begin{abstract}
A two-dimensional kinetic model has been used to parametric investigation of the spark-type partial discharge pulses inside the micro-cavities. The model is based on particle-in-cell methods with Monte Carlo Collision techniques for modeling of collisions. Secondary processes like photo-emission and cathode-emission are considered. The micro-cavity may be sandwiched between two metallic conductors or two dielectrics. The discharge within the micro-cavity is studied in conjunction with the external circuit. The model is used to successfully simulate the evolution of the discharge and yield useful information about the build-up of space charge within the micro-cavity and the consequent modification of the applied electric field. The phase-space scatter plots for electrons, positive, and negative ions are obtained in order to understand the manner in which discharge progresses over time. The risetime and the magnitude of the discharge current pulse are obtained and are seen to be affected by micro-cavity dimensions, gas pressure within the micro-cavity, and the permittivity of surrounding dielectrics. The results have been compared with existing experimental, theoretical, and computational results, wherever possible. An attempt has been made to understand the nature of the variations in terms of the physical processes involved.
\end{abstract}

Keywords Partial discharges - Submillimeter cavities . PIC-MCC simulation · Spark-type discharges

Alireza A. Ganjovi

alirezaganjovi@yahoo.com; ganjovi@kgut.ac.ir

1 Photonics Research Institute, Institute of Science, High Technology \& Environmental Sciences, Graduate University of Advanced Technology, Kerman, Iran

\section{Introduction}

Some of the principal factors that can influence partial discharges (PD's) and other such discharges inside micro-cavities are the dimension of micro-cavity, the pressure of the gaseous environment and gas composition, the magnitude and frequency of the applied voltage, the electrode arrangement and geometry, and the properties, condition, and age of the insulation. The different character of the discharges affects in turn the response of conventional PD pulse detectors, which is dependent to the pulse shape and its rise-time [1-3].

The rise-time of PD pulses is one of the most important subjects. The rise-time of the discharge pulses determines the type of the PD detection circuit that must be employed to the detection of the discharge pulses and record their pulse shape attributes with specified precision and accuracy [4]. While it is evident that the rise-time of the detected PD pulses is depend upon the bandwidth of the discharge detection equipment as well as location of PD source, the rise-time is also very much dependent to the discharge mechanism as such and the availability of free electrons to initiate that [4]. In contrast to the long rise-times characterizing the discharge pulse by glow discharges, spark-type discharges exhibit typically short rise-times and large magnitude pulses [2, 4]. Bartnikas et al. [2] studied the effect of over-voltage on PD rise-time. They reported that with greater over-voltages, the PD pulses assume shorter rise-times and pulse widths as well as increasing magnitudes, thereby inferring larger charge transfers.

It has been demonstrated earlier that the discharge mechanisms in a micro-cavity are essentially of the Townsend type and the form of the ensuing discharge current pulse at the time of a spark-type breakdown is controlled by the electric field created by the ion space charge in the micro-cavity [1]. 
A few studies deal with cavities in the submillimeter range; these include modeling of avalanches inside cylindrical micro-cavities by Burgener et al. [5] and experimental studies by $\mathrm{Gu}$ et al. [6]. As already reported in the literature, in large disk-shaped voids, transition takes place from streamer mechanism to Townsend mechanism due to a conducting layer formed on the dielectric walls by discharge by-products [7-11]. However, an important point to note is that streamer discharges do not occur in submillimeter cavities, in the absence of the critical length required for formation of a streamer head [1].

Kinetic PIC-MCC simulation methods are known to be better suited for problems in which the particle distribution may deviate significantly from Maxwellian, such as when trapping or particle stochastic heating occurs [12-14]. Additionally, the PIC-MCC method guarantees high accuracy for both transient and stationary problems [15], and yields more detailed information about the evolution of the discharge. Radjenovic et al. [16, 17] used a one-dimensional PIC-MCC model to study Paschen's law for gas breakdown at small electrode separations.

A two-dimensional kinetic model based on PIC-MCC technique to study the behavior of spark-type PDs inside the micro-cavities has been developed [18]. The same model was used to investigate the filamentary distribution of spark-type PD's inside a micro-cavity [19]. In another work, a radiation transport (RT) model integrated with the PIC-MCC model was developed to study the behavior of excited (radiative) states of air within discharge in the narrow channel [20]. The radiative state atoms were described by a fluid model combined with the HolsteinBiberman equation.

Using this model, in this paper, the influences of different parameters of micro-cavity on the different features of a spark-type PD pulse such as discharge current, current rise-time, etc., with two different electrode configurations, i.e., metallic-metallic (MM) and dielectric-dielectric (DD) are examined [18]. The model has been employed for performing an extensive parametric study relevant to effect of quantities such that micro-cavity dimensions, gas pressure inside the micro-cavity, and dielectric permittivity on the PD development within MM and DD micro-cavity. The intent is to discuss the results obtained systemically using the model to establish the effect of above quantities on the nature and shape of PD pulses in micro-cavities undergoing a spark-type discharge in air.

\section{Computational techniques}

In the PIC scheme, particles are defined in a continuum position and velocity space. Field values are defined at discrete locations in space. Particle and field values are advanced sequentially in time, starting from initial conditions. The particle equations of motion are solved at every time-step, using field values interpolated from the discrete grid to particle locations. The force on every particle is computed by interpolation of the field values from the grid position to the given particle positions. The position and velocities of each particle is next updated based on the solution of the classical equation of motion [21]. Next, particle boundary conditions are applied. For modeling collisions, the Monte Carlo collision (MCC) scheme is applied [22]. Source terms for the field equations are accumulated from the particle locations to the grid locations. The field values are then advanced by one time-step, and the time loop starts again (Fig. 1).

In the PIC-MCC scheme, each super-particle, representative of one or a much larger number of real particles, is designated in continuum space by its position and velocity. Initially, a Maxwellian distribution is assumed for electrons and ions. Further, the particles are uniformly distributed in the gap space. Particle boundary conditions such as absorption and emission are used to account for the relation between the discharge current in the gap and the current in the external circuit. When an electron or ion passes from the discharge into an end wall, it adds to the wall charge and is deleted from the list of active particles [13]. Additionally, secondary emissions occur when a charged particle impacts a surface (metallic or dielectric) causing ejection of electrons from the surface.

In the current work, the gas within the cavity is assumed to be air at atmospheric pressure. Therefore, photo-emission at the cathode is likely to be the dominant secondary

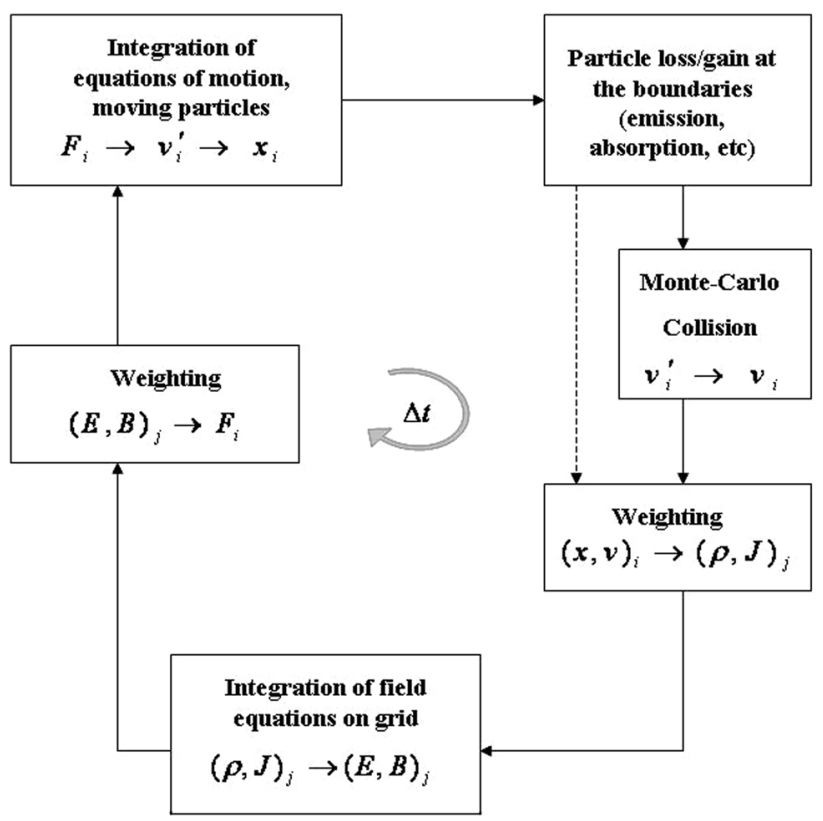

Fig. 1 Schematic representation of the computation sequence for particle in cell-Monte Carlo (PIC-MCC) method 
emission process, rather than electron emission from cathode or anode due to ion or electron bombardment. However, all of the above processes have been accounted for in the model. After emission of a new electron, its energy is also assigned from an assumed Maxwell-Boltzmann distribution. The interaction of charged particles with neutral atoms and molecules, and other collisional processes are included using a Monte Carlo Collision technique [12]. Three main charged particle species, viz. electrons, positive ions, and negative ions, have been considered. The major electron-neutral molecule impact reactions included are (1) elastic, (2) excitation, (3) ionization (including all important ionization reactions), and (4) electron attachment. Related cross-sectional values are extracted from experimental data available in the literature [23]. Integral cross-section values for electron interaction in dry air have been determined from its molecular constituents by assuming a target composition of $78.09 \% \mathrm{~N}_{2}$ and $21.19 \% \mathrm{O}_{2}$ [24]. Only elastic and charge transfer collisions between ions and neutrals are considered. Because ion-molecule cross-sections are not readily available, a constant cross-section $\left(10^{-20}\right)$ has been assumed based on a hard-sphere collision model [25].

A significant contribution of this work is that the discharge within the cavity is simulated in conjunction with an external circuit comprising of a voltage source, and a blocking capacitor that stops the flow of any average current. The coupling between the charge flow within the micro-cavity and the external current is obtained via the parallel-plane electrodes, which may be metallic or dielectric, depending on the location of the discharge site.

The potentials and fields are obtained using Poisson's equation:

$\nabla \cdot \varepsilon_{0} \nabla \Phi(x, t)=\rho(x, t)$.

The potential $(\Phi)$ can be separated as follows $[12,26$, 27]:

$\Phi=\Phi_{\mathrm{P}}+\sum_{\text {Boundaries }} \Phi_{\mathrm{L}}$

where $\Phi_{\mathrm{P}}$ and $\Phi_{\mathrm{L}}$ represent the Poisson and Laplacian parts of electrical potential $(\Phi)$.

Therefore, the field may be described by the combination of the following equations:

$$
\begin{aligned}
& \nabla \cdot \varepsilon_{0} \nabla \Phi_{\mathrm{P}}=-\rho, \\
& \nabla \cdot \varepsilon_{0} \nabla \Phi_{\mathrm{Li}}=0 .
\end{aligned}
$$

The boundary condition for Eq. (3) is $\Phi=0$ on all boundaries as the Poisson field is solely due to charge in the medium. For each boundary with a Dirichlet condition, Eq. (4) is solved for $\Phi_{i}=0$ on the equi-potential surface, and $\Phi=0$ elsewhere which gives us $\Phi_{\mathrm{Li}}$ (the potential due to the ith Laplacian field). $\Phi_{\mathrm{L}}$ is obtained by the superposition of all the Laplacian fields. Neumann boundary conditions are included through $\Phi_{\mathrm{P}}=0$. This method neglects charges induced by a driven electrode on other boundaries which are connected to an external circuit. It is also possible to solve the field Poisson equation with boundaries and circuits [12, 21, 28].

In this work, identical cavities, as might occur at discharge sites, with two different electrode configurations have thus been considered. These are the two generic cases described below. The model is two-dimensional with Neumann boundary conditions on the side-walls. Dirichlet boundary conditions (potential drop specified) are assumed on the electrodes.

\section{Metallic-metallic configuration}

The first case considers a cavity between MM electrodes. For this configuration, the potential at the anode can be obtained as follows [29]:

$$
\begin{aligned}
\varphi_{0}^{t} & =\left(\frac{\varepsilon_{0}}{\Delta x} N+C / A\right)^{-1} \times\left(\sigma_{\mathrm{T}}^{t-1}+\frac{1}{A}\left(C V(t)-Q_{\mathrm{c}}^{t-1}+Q_{\mathrm{Conv}}^{t}\right)\right. \\
& \left.+\frac{\Delta x}{2} \sum_{j=0}^{j=N-1} \rho_{0 j}+\frac{\varepsilon_{0}}{\Delta x} \sum_{j=0}^{j=N-1} \varphi_{1 j}^{t}\right)
\end{aligned}
$$

where $\sigma_{T}, A, C, V(t), Q_{\mathrm{c}}, Q_{\mathrm{r}}, Q_{\mathrm{conv}}, \rho, \varphi_{i j}$, and $N_{j}$ are, respectively, the total surface charge density on the electrodes at a given time ' $t$ ', electrode cross-sectional area, capacitance of the blocking capacitor, driving voltage, charge in the external circuit, charge convection due to discharge, space charge density within the discharge, the electric potential at each grid point, and the number of grid divisions along the $y$-axis.

\section{Dielectric-dielectric configuration}

The second case we implement is that of DD where the micro-cavity is bounded in both sides by dielectrics. This necessitates the incorporation of a second dielectric slab between the metallic cathode and the void wall at the cathode end (Fig. 2a). The equivalent circuit is shown in Fig. $2 \mathrm{~b}$, where $C_{\mathrm{g}}, C_{\mathrm{d} 1}$, and $C_{\mathrm{d} 2}$ represent the equivalent capacitances of the micro-cavity and the dielectric layers, respectively. Assuming $C_{\mathrm{d} 1}=C_{\mathrm{d} 2}=C_{\mathrm{d}}$, using $\mathrm{KCL}$, it is possible to obtain the following relations:

$C_{\mathrm{d}} \frac{\mathrm{d} U_{\mathrm{d}}}{\mathrm{d} t}=I_{\mathrm{ext}}$,

$A \frac{\mathrm{d} \sigma_{\mathrm{s}}}{\mathrm{d} t}=I_{\mathrm{ext}}+A J_{\mathrm{conv}}$,

$V_{\mathrm{c}}=V(t)-2 U_{\mathrm{d}}-\varphi_{0}$,

where $U_{\mathrm{d}}, I_{\mathrm{ext}}, \sigma_{\mathrm{s}}, J_{\text {Conv }}$, and $V_{\mathrm{c}}$ are, respectively, the potential across each dielectric slab, total external current, 

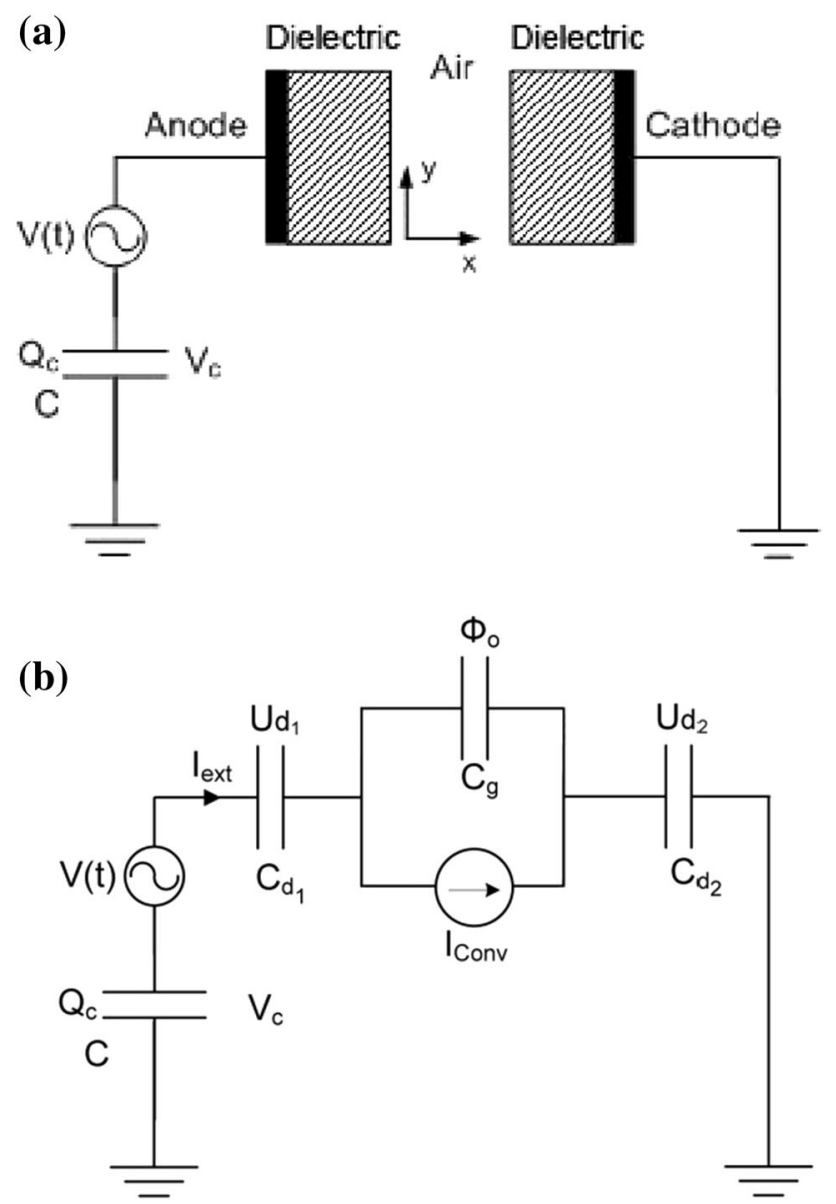

Fig. 2 a Schematic representation of a dielectric-dielectric microcavity; the external circuit consists of a voltage source and a blocking capacitor which stops the flow of any average current, b the corresponding equivalent circuit

charge density on the dielectric surface, discharge current density $\left(I_{\text {conv }}=I_{\mathrm{d}}=A J_{\text {Conv }}\right)$, and voltage across blocking capacitor. By combining the above equations, Eq. (5) may be rewritten for DD case as follows

$$
\begin{aligned}
\varphi_{0}^{t}= & \left(\sigma_{\mathrm{s}_{\mathrm{T}}}^{t-1}+\frac{1}{A}\left(\alpha C V(t)-\alpha Q_{\mathrm{c}}^{t-1}-2 \alpha C U_{\mathrm{d}}^{t-1}+Q_{\text {Conv }}^{t}\right)\right. \\
& \left.+\frac{\Delta x}{2} \sum_{j=0}^{j=N} \rho_{0 j}+\frac{\varepsilon}{\Delta x} \sum_{j=0}^{j=N} \varphi_{1 j}^{t}\right) \cdot\left(\frac{\varepsilon}{\Delta x} N+\alpha C / A\right)^{-1},
\end{aligned}
$$

where $\alpha=\left(1+2 C / C_{\mathrm{d}}\right)^{-1}$. The derivation of Eq. (9) is presented in "Appendix" section.

\section{Simulation results}

This section is devoted to the presentation of the results obtained by numerical modeling. A grid-size of $\Delta x=\Delta y=2 \times 10^{-6} \mathrm{~m}$, and a time-step of $\Delta t=10^{-14} \mathrm{~s}$ are used; typical simulation times of $24 \mathrm{~h}$ are required with a dual processor SUN workstation. As a first step, in this section, we trace the evolution of the distribution of charged particles within the submillimeter void.

The scatter plots in phase space for velocities in $x$ direction i.e., $\left(V_{x}, x, y\right)$ for electrons and positive ions in Figs. 3 and 4 at particular instants of time. As shown here, drift velocity of electrons formed during the discharge is much higher than ions $\left(10^{3}\right.$ times) and quickly traverse the micro-cavity. All charged particles are distributed about the center-line $y=50 \mu \mathrm{m}$. Most of the charged particles are created very close to the anode. In the early stages, positive ions are distributed evenly over the inter-electrode distance, except for a small region near the cathode. It is also uniformly distributed along $y$-direction. The number of ions near the anode increases by ionization with passage of time. The positive ions slowly drift toward the cathode, till at about $13 \mathrm{~ns}$, the entire gap distance is almost evenly populated. From this time onward, ions gradually leave the micro-cavity, thus depleting the ions resident in the gap. This is similar to the evolution of the electrons within the gap, as seen in Fig. 3. Electrons move faster and travel only the distance between the ionization region and the anode. As is clear from Fig. 4, the ion velocities increase as they reach near the cathode, accelerated by the electric field.

In following, the effects of micro-cavity dimensions, gas pressure, and dielectric permittivity on the PD pulse features such as discharge current magnitude and its rise-time have been investigated. Obviously, not all PD pulses are characterized by very a rapid rise-time; with a high probability of occurrence of a free electron within the gas of occluded cavities, only small or moderate over-voltages may be required to precipitate a breakdown event. Unless otherwise mentioned, all simulations reported in this work are performed for operating conditions specified in Table 1.

\section{Micro-cavity length}

For a relatively short anode-cathode gap distance, the effect of the micro-cavity dimensions on the discharge characteristics, from experimental as well as theoretical findings, is evident [7, 30-41]. In this paper, the length of the micro-cavity is varied from 50 to $600 \mu \mathrm{m}$, while the applied electric field across micro-cavity is held constant at $E=10^{6} \mathrm{~V} / \mathrm{m}$. As the length of the micro-cavity increases, for a given pressure, the residence time of ionizing particles inside the micro-cavity is higher. Each electron suffers a larger number of ionizing collisions as it travels from the cathode to the anode; as a result, the production of charged particles inside the micro-cavity is much higher at higher lengths. This is evident as peak value of charged particles 

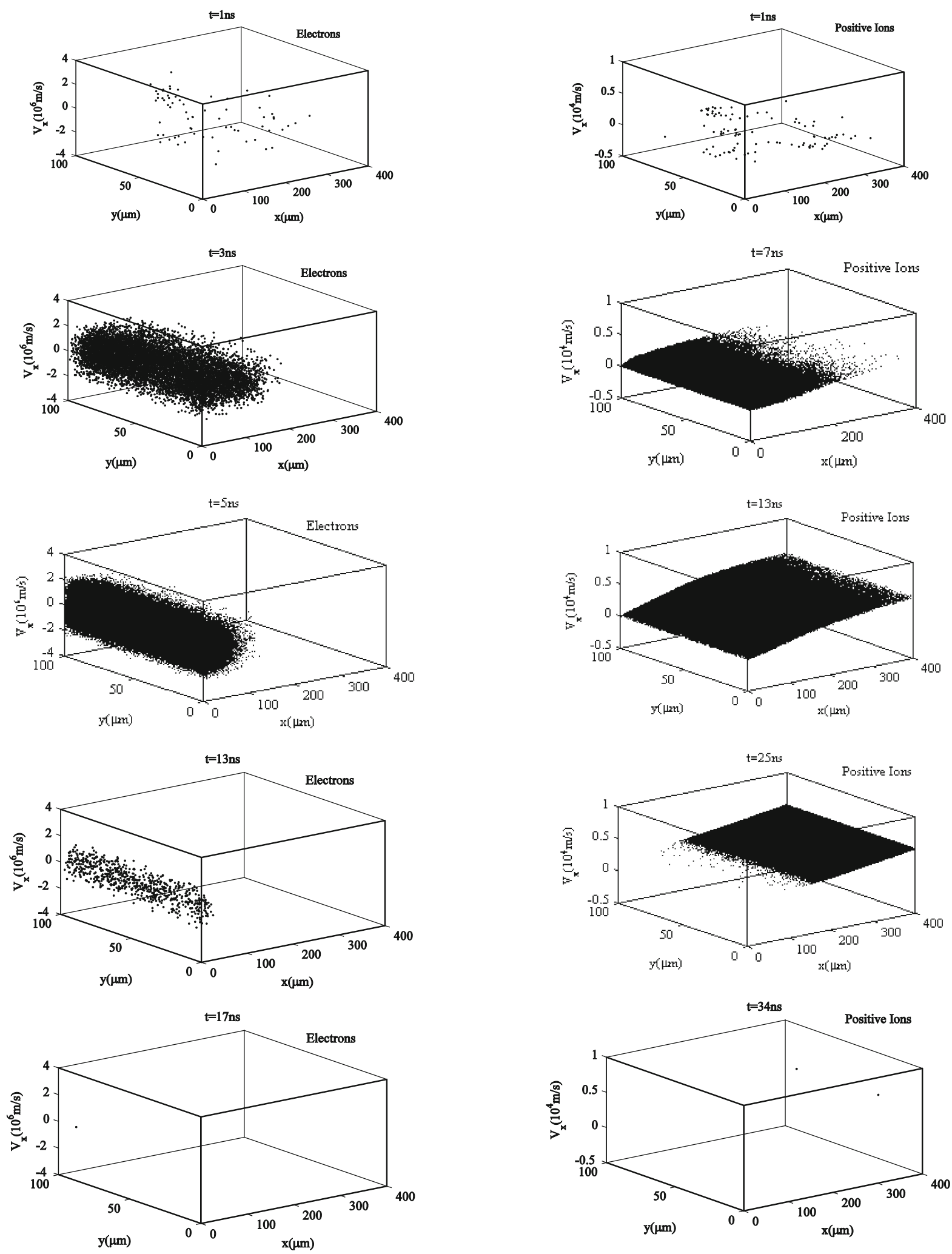

Fig. 3 Scatter plot of electrons in the phase-space $\left(V_{x}, x, y\right)$ inside the micro-cavity at particular instants of time; $L_{\mathrm{X}}=400 \mu \mathrm{m}, L_{\mathrm{y}}=100$ $\mu \mathrm{m}, L_{\mathrm{z}}=100 \mu \mathrm{m}$, applied electric field across the micro-cavity: $E=1.292 \times 106 \mathrm{~V} / \mathrm{m}$, gas pressure: $P_{\mathrm{g}}=760$ Torr

Fig. 4 Scatter plot of positive ions in the phase-space $\left(V_{y}, x, y\right)$ inside the micro-cavity at particular instants of time; $L_{\mathrm{X}}=400 \mu \mathrm{m}$, $L_{\mathrm{y}}=100 \mu \mathrm{m}, L_{\mathrm{z}}=100 \mu \mathrm{m}$, applied electric field across the microcavity: $E=1.292 \times 106 \mathrm{~V} / \mathrm{m}$, gas pressure: $P_{\mathrm{g}}=760$ Torr 
Table 1 Simulation data

\begin{tabular}{lll}
\hline Property & Symbol & Value \\
\hline Micro-cavity length & $L_{\mathrm{X}}$ & $200 \mu \mathrm{m}$ \\
Micro-cavity width & $L_{\mathrm{y}}$ & $100 \mu \mathrm{m}$ \\
Micro-cavity depth & $L_{\mathrm{z}}$ & $100 \mu \mathrm{m}$ \\
Dielectric thickness & $L_{\mathrm{d}}$ & $200 \mu \mathrm{m}$ \\
Background gas pressure & $P_{\mathrm{g}}$ & $101,325 \mathrm{~Pa}$ \\
Background gas temperature & $T_{\mathrm{g}}$ & $0.026 \mathrm{eV}$ \\
External capacitor & $C$ & $40 \mathrm{pF}$ \\
Initial number of charged particles & $n_{\mathrm{f}}$ & 10 \\
Photo-emission coefficient & $\lambda_{\mathrm{ph}}$ & $5 \times 10^{-5}[30]$ \\
Ion-induced emission coefficient & $\lambda_{\mathrm{i}}$ & $1.5 \times 10^{-3}[30]$ \\
Dielectric constant of air & $\varepsilon_{0}$ & $8.85 \times 10^{-12} \mathrm{~F} / \mathrm{m}$ \\
\hline
\end{tabular}
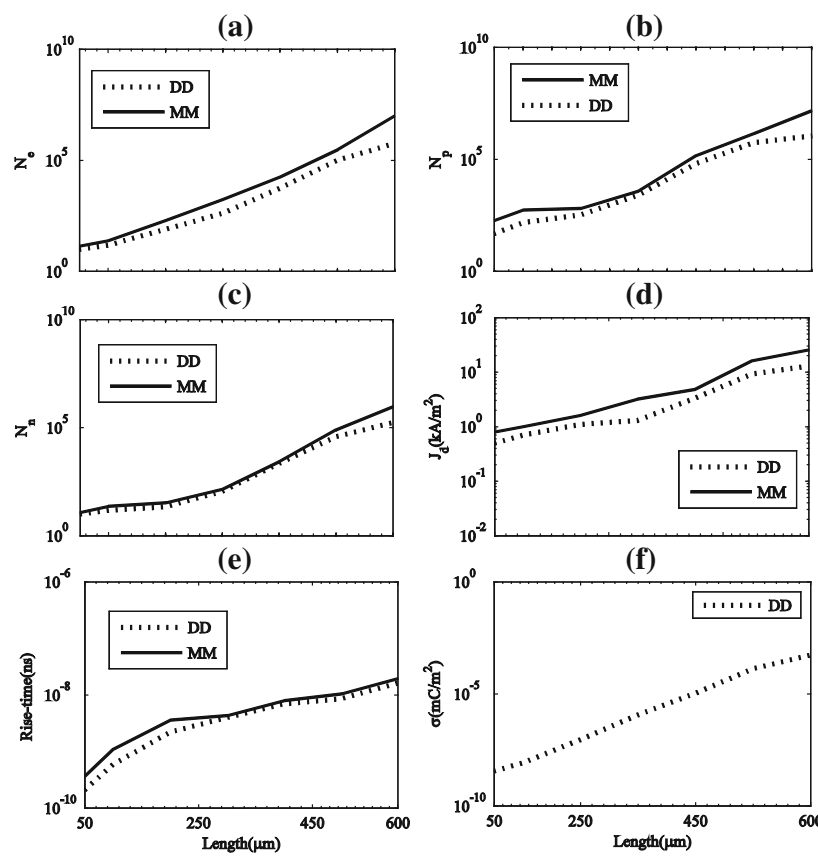

Fig. 5 Variation with micro-cavity length of a number of electrons, b number positive ions, c number of negative ions, d discharge current, e current rise-time, for both MM and DD configurations, and f the magnitude of density of charge accumulated on the dielectrics

in Fig. 5a-c. Results obtained by Serra et al. similarly show that the increase in length of micro-cavity results in increase in the number of electrons generated inside the micro-cavity [41]. Aging and life models by Montanari and coworkers $[34,35]$ have similarly shown that the number of electrons generated inside the cavity increases with cavity length. The electrons generated inside the cavity are the main carriers of PD current [40, 41]. Increase in the number of charged particles is reflected in an increase in the magnitude of discharge current shown in Fig. 5d. This finding is in agreement with experimental results reported by Devins [33], which shows that the discharge current is sensitive to length of micro-cavity, increases with microcavity length. Bartnikas [32] varied gap length from $0.1 \mathrm{~mm}$ to $1 \mathrm{~mm}$ and reports that a reduction in the gap length results in the disappearance of the pulse discharge (spark-type) and the onset of a diffused glow (pseudo-glow and glow discharge).

Figure 5e shows the variation of PD pulse current risetime as a function of micro-cavity length. The rise-time increases as length increases. This is understandable as the ionization process in longer micro-cavities require a longer time to reach peak values. These finding corroborates the experimental results by Morshuis [36]. He studied the pulse shape of PD in disk-shaped cavities in polyethylene and other polymers. He varied the length of the cavities between $30 \mu \mathrm{m}$ and $3.7 \mathrm{~mm}$. He reports that for cavities with a diameter/length ratio $\gg 1$, PD rise-time is lower [7, 40]. Ramachandra et al. measured fast discharge pulses with rise-times below $1 \mathrm{~ns}$ [42] for void depths (lengths) of 80,120 , and $200 \mu \mathrm{m}$. His results show that discharge current rise-times are faster for smaller void depths. Experimental results by Wetzer et al. for PD waveforms show a longer rise-time and pulse duration for micro-cavities (submillimeter) with greater lengths [37].

The variation of charge accumulation at the dielectric anode and cathode at the end of discharge pulse is shown as a function of micro-cavity length in Fig. 5f. The amount of accumulated charge density is calculated as the difference between the charges at the beginning and end of discharge, $\left(\Delta \sigma_{\mathrm{s}}=\sigma_{\mathrm{si}}-\sigma_{\mathrm{sf}}\right)$. As larger numbers of charged particles are deployed in the discharge with increased length, the amount of charge accumulated on the dielectric surfaces also increases. This value is always negative for the anode, as the absorbed negative charges lower the applied potential on the anode. For the MM case, the accumulated charge is soon dissipated into the external circuit, and there is no charge accumulation at discharge extinction.

Figure 6 depicts the number of charged particles for both $\mathrm{MM}$ and DD configurations and for two different microcavity lengths. This is in agreement with simulation results obtained by Novak et al. [43] using a fluid model, for a 0.5$\mathrm{mm}$ gap and DD configuration. Negative ions are depleted fast. As is seen here, the number of negative ions is much lower than that of electrons or positive ions. For the DD configuration, a smaller number of charged particles are involved. Interestingly, for the smaller micro-cavity length, the rise-time for the DD is smaller, and so is the fall-time.

The time evolution of the anode potential for different micro-cavity lengths, $\varphi_{0}$, is presented in Fig. 7a, b. A similar decrease in anode potential with time was observed by Bartnikas et al. for $0.5 \mathrm{~mm}$ gap and DD configuration. It may be noted that the effect of external circuit is ignored by Bartnikas et al. [1]. The values remain constant with time for MM configuration as expected, because the 
(a)

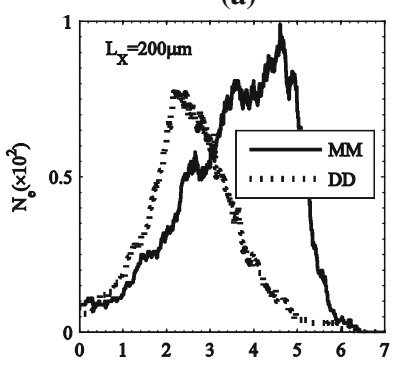

(c)

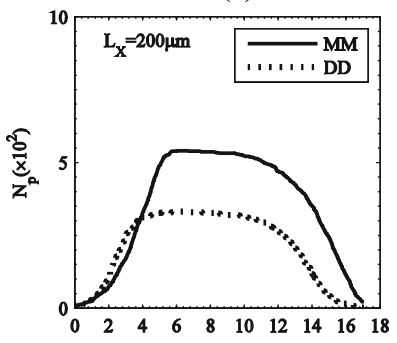

(e)

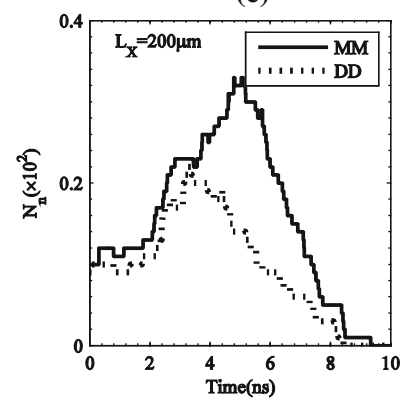

(b)

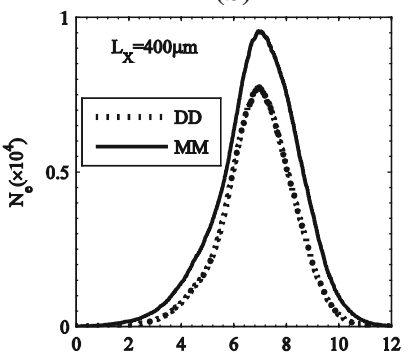

(d)

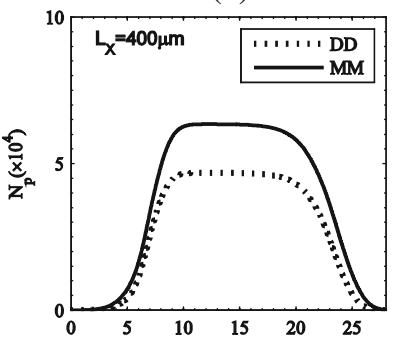

(f)

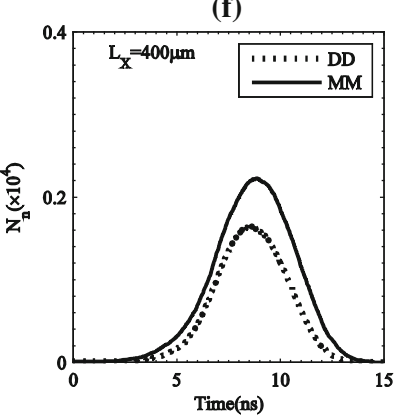

Fig. 6 Time history of (a and b) the number of electrons, (c and d) the number of positive ion, (e and $\mathbf{f}$ ) the number of negative ions for two different micro-cavity lengths: $L_{\mathrm{X}}=200 \mu \mathrm{m}$ and $L_{\mathrm{X}}=400 \mu \mathrm{m}$

conductors are maintained at a constant applied potential by the external circuit. However, for DD configuration, due to the role of dielectric on the electric field next to dielectric anode in the discharge medium and also initially presence of positive-charged particles in this region, the dielectric anode electrode potential first increases. Then, because of accumulation of negative-charged particles (i.e., electrons and negative ions) on anode dielectric, the anode potential decreases, settling at considerably decreased values at the end of the discharge-period. The anode dielectric potential, for DD configuration, at the end of the discharge $\left(\varphi_{0 \mathrm{f}}\right)$ for micro-cavity length of $200 \mu \mathrm{m}$ is reduced to $99.9 \%$ of its initial value $\left(\varphi_{0 \mathrm{i}}\right)$ and for length of $400 \mu \mathrm{m}$, it has reached to $97 \%$ of its initial value. Thus, while the applied electric field across micro-cavity is kept constant at different lengths, the $\operatorname{drop}\left(\Delta \varphi_{0}=\varphi_{0 i}-\varphi_{0 \mathrm{f}}\right)$ at higher micro-cavity lengths is higher.

The change in potential is brought about by charge accumulation at the dielectric electrodes, and as it is clear in Fig. $7 \mathrm{c}, \mathrm{d}$, the charge accumulated on the dielectrics for

(a)

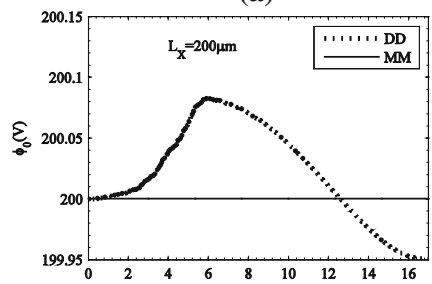

(c)
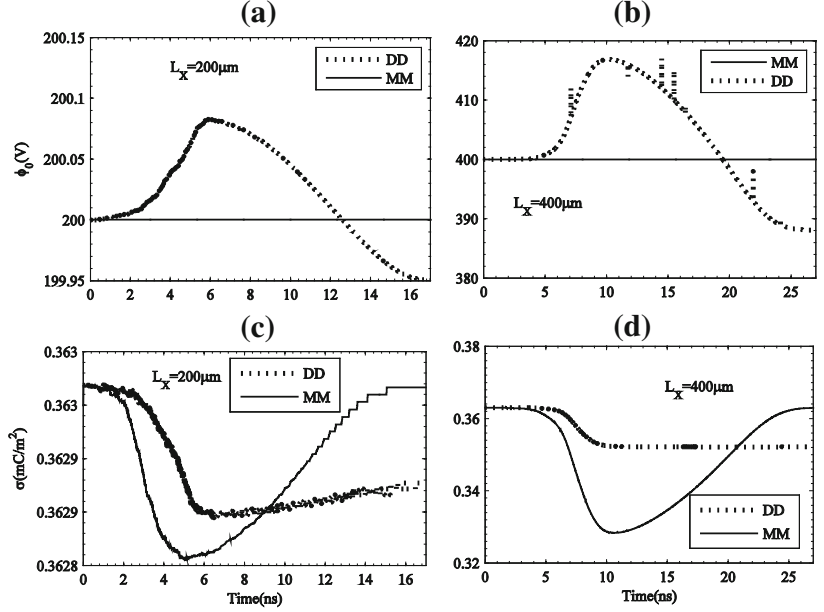

(d)

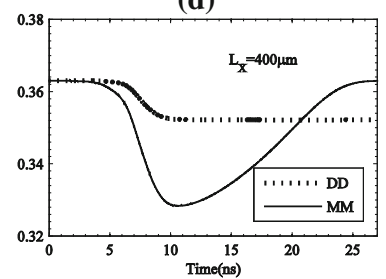

Fig. 7 Time history of the electric potential on the anode dielectric for a length of $L_{\mathrm{X}}=400 \mu \mathrm{m}$, b length of $L_{\mathrm{X}}=400 \mu \mathrm{m}$, and the charge density on the anode dielectric for c length of $L_{\mathrm{X}}=400 \mu \mathrm{m}$, d length of $L_{\mathrm{X}}=400 \mu \mathrm{m}$

$200-\mu \mathrm{m}$ micro-cavity length $\left(10^{-4} \mathrm{mC} / \mathrm{m}^{2}\right)$ is much $<400 \mu \mathrm{m}$ micro-cavity length $\left(0.01 \mathrm{mC} / \mathrm{m}^{2}\right)$. Nikonov presented the variation of the field at the anode with time [44], and this shows a trend similar to change in $\sigma$. It may be noted that $\sigma=\varepsilon_{0} E_{\text {anode }}$, where $E_{\text {anode }}$ is the field at the anode, and $\varepsilon_{0}$ the permittivity of free space. The variation of $E_{\text {anode }}$ in Nikonov's work may therefore be taken to be a corroboration of the general trend in variation of surface charge density accumulated on the anode surface.

It is interesting to study the effect of length on the PD pulse by looking at the evolution of the electric field inside the micro-cavity. Figure 8 shows the variation in the axial field (component in the direction of the field) along the micro-cavity length, at different moments of time. As may be seen, the field within the micro-cavity is originally uniform for both lengths i.e., $L_{\mathrm{X}}=200 \mu \mathrm{m}$ and $L_{\mathrm{X}}=400 \mu \mathrm{m}$. Due to less strength of ionization at lower micro-cavity lengths, the effect of space charge on the applied electric field in case of $L_{\mathrm{X}}=200 \mu \mathrm{m}$ is much less than $L_{\mathrm{X}}=400 \mu \mathrm{m}$ (Fig. 8a, b). Wu et al. [45] showed that as length of micro-cavity increases, the effect of the field due to charged particles inside the micro-cavity becomes more evident. Generally, for the MM case, the field at the dielectric anode is reduced considerably within a very short time. Positive charges take longer to travel the gap distance, and a positive space charge region forms ahead of the anode where they accumulate. This results in a negative field ahead of the anode. The field in the rest of the microcavity is low and positive, being directed from the positive space charge region toward the cathode. As positive ions gradually travel toward the cathode, the field in the near vicinity of the anode is relieved and gradually reaches initial values. Simultaneously the field near the cathode is enhanced in the direction of the applied electric field, due 
(a)

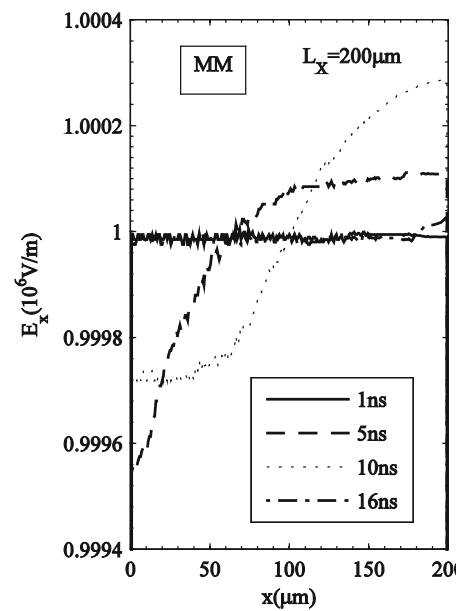

(c)
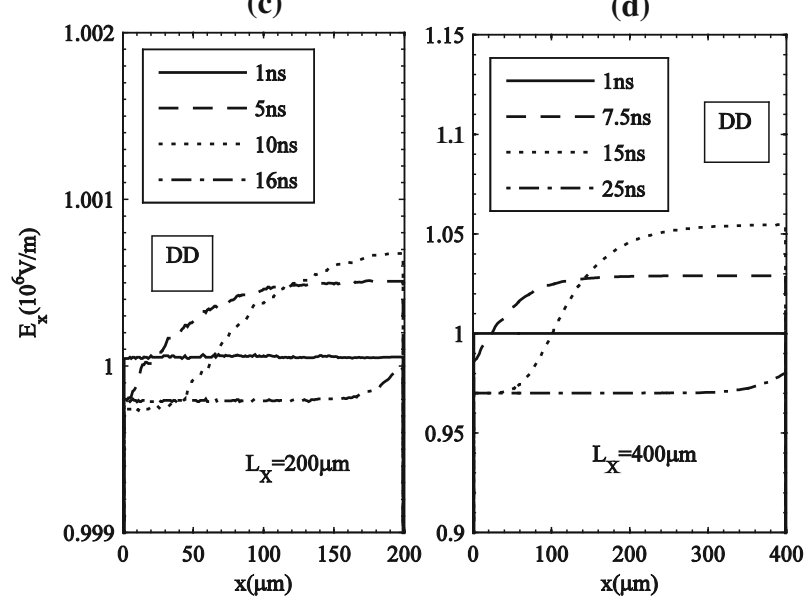

Fig. 8 Variation of $E_{\mathrm{x}}$ along micro-cavity length at different times for different configurations at two different values of micro-cavity length

to the positive charge cloud nearing the negatively charged cathode. Ultimately after $25 \mathrm{~ns}$ (for length of $L_{\mathrm{X}}=400$ $\mu \mathrm{m})$, with the gap being cleared of all charges, original field values are regained throughout the micro-cavity.

For the DD case, shown in Fig. 8c, d, the evolution of the field values is similar to the previous case till about $6 \mathrm{~ns}$. However, the field at the anode never recovers the original values owing to accumulation of negative charges at the anode, lowering the potential thereof. Here too, the field within the micro-cavity becomes uniform; the ultimate field value is, however, lower than in the MM case. Again due to higher effect of space charges at higher micro-cavity lengths, change in the applied electric field is much higher at higher micro-cavity lengths. This is because of the space charge field (produced due to negative charge accumulation at the dielectric anode) opposes the applied field.

Figure 9 presents the electric field $\left(E_{\mathrm{x}}\right)$ as a function of time at different axial distances from the anode (along the (a)

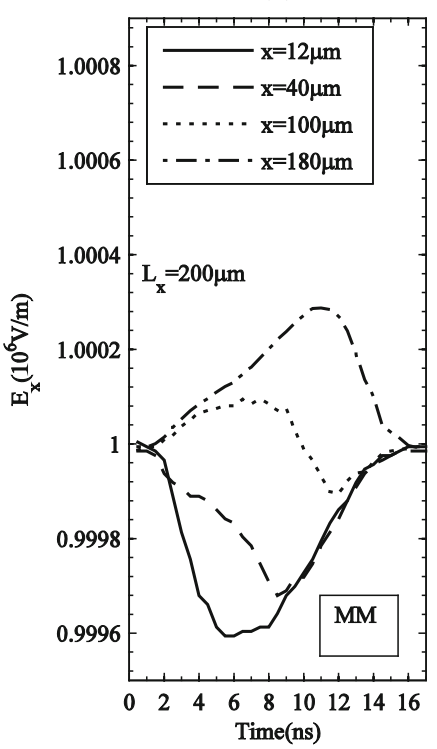

(c)

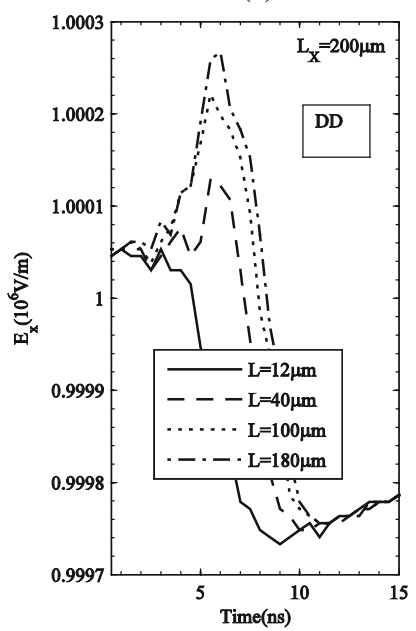

(b)

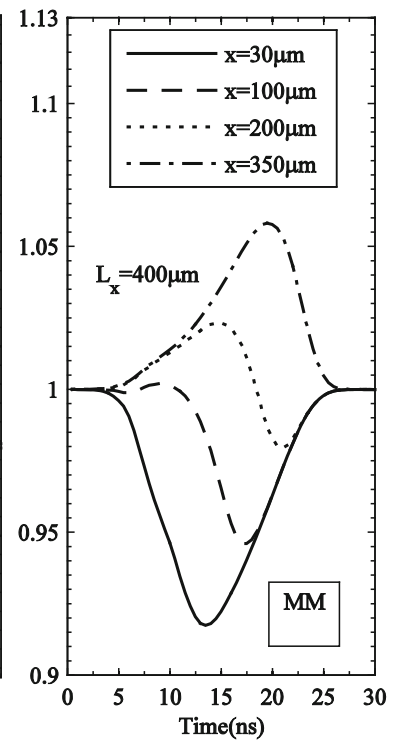

(d)

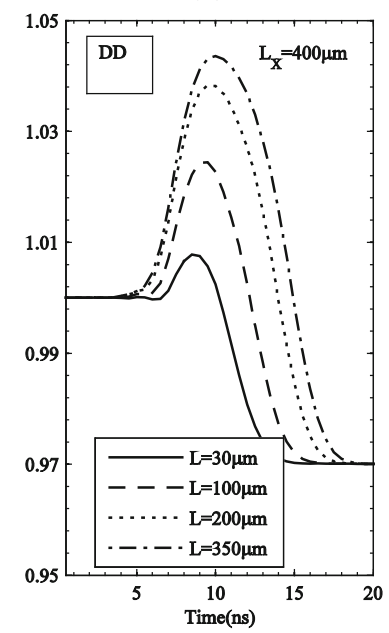

Fig. 9 Time history of $E_{\mathrm{x}}$ as a function of time at different points along $\mathrm{x}$-axis for different configurations for two different values of pressure

length of the micro-cavity) for two lengths of micro-cavity i.e., $L_{X}=200 \mu \mathrm{m}$ and $L_{X}=400 \mu \mathrm{m}$. In the MM case, the field variation is similar in both cases; the magnitudes though are different, the field level near the anode falls initially, followed by a rise which is due to lowering of field values owing to an initial positive charge accumulation in front of the anode electrode. While the electric field near the cathode is almost close to its initial value, the electric field near the anode is at a minimum (at $t=6 \mathrm{~ns}$ ) when most of the negative charges have left the microcavity. After this moment, the breakdown mechanism becomes much more space charge controlled, and the electric field near the cathode will be under influence of the positive charges arriving in its vicinity and consequently 
increases. At distances near the cathode, there is a considerable enhancement in the electric field between the positive ions and cathode. Similar observations were made by Serdyuk et al. in modeling of electric discharges in a 3-mm air-gap between parallel plate solid dielectric barriers [46]. They showed that the interaction of the avalanche with the solid dielectric led to charging of the barriers.

Optical studies by Morshuis et al. revealed a large number of separate discharging sites dependent on the void length $[7,40]$. They have reported that the current pulses of these separate discharges were integrated by the relatively slow detector, resulting in extremely high discharge values. However, in this work, it has been assumed that only one discharging site on dielectric anode (DD configuration).

Thus, it could be concluded that greater PD pulse current rise-time and charge accumulation on anode and cathode dielectric surfaces occurs at higher micro-cavity lengths.

\section{Micro-cavity width}

It has been shown that on increasing the micro-cavity width, there is a greater likelihood of movement of charge carriers in the direction perpendicular to applied electric field ( $y$-direction) $[18,19]$. This results in increased dissipation of energy of the PD pulse. Ionization and production of new charged particles therefore, reduces, as was seen in the peak values of charged species.

Figure 10 shows the PD pulses for MM and DD configurations for two different micro-cavity widths. For a width of $L_{\mathrm{y}}=300 \mu \mathrm{m}$, the PD pulse current peak occurs at 350 and $300 \mu \mathrm{A}$ for $\mathrm{MM}$ and DD configurations, respectively. For a width of $L_{\mathrm{y}}=600 \mu \mathrm{m}$, the values for PD (a)

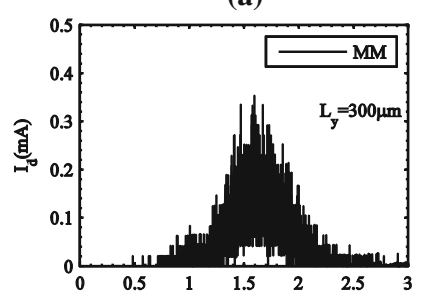

(c)

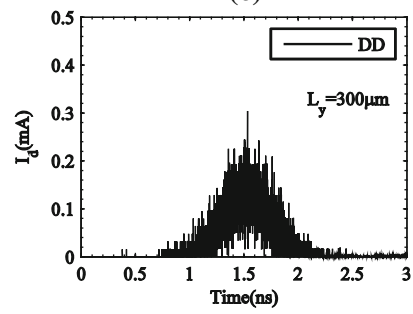

(b)

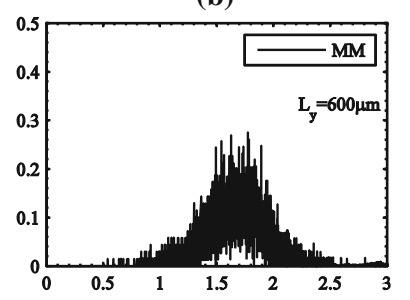

(d)

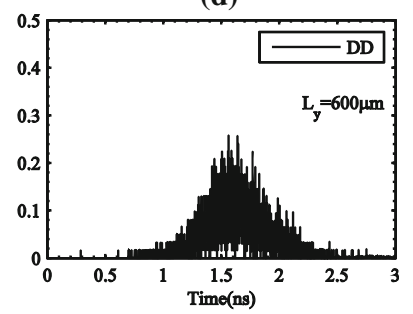

Fig. 10 Time history of PD pulse current for a MM configuration for width of $L_{\mathrm{y}}=300 \mu \mathrm{m}, \mathbf{b} \mathrm{MM}$ configuration for width of $L_{\mathrm{y}}=600$ $\mu \mathrm{m}$, c DD configuration for width of $L_{\mathrm{y}}=300 \mu \mathrm{m}$, and d DD configuration for width of $L_{\mathrm{y}}=600 \mu \mathrm{m}$ pulse current occur at 273 and $256 \mu \mathrm{A}$ for MM and DD configurations which are lower than the corresponding values for the case of $L_{\mathrm{y}}=300 \mu \mathrm{m}$. Thus in the wider micro-cavity, reduced peak values are observed. The current is lower for a DD configuration. The variation in micro-cavity width does not affect the resultant PD pulse current shape. Gu et al. varied the micro-cavity diameter in the range of 50-600 $\mu \mathrm{m} \mathrm{[6]} \mathrm{and} \mathrm{found} \mathrm{that} \mathrm{the} \mathrm{ionization}$ coefficient increases rapidly as micro-cavity diameter decreases. This is in agreement with the presented results in this paper.

Figure $11 \mathrm{a}, \mathrm{b}$ shows the time evolution of the anode potential, $\varphi_{0}$, for different micro-cavity widths. The anode dielectric potential, for DD configuration, at the end of the discharge for micro-cavity width of $300 \mu \mathrm{m}$ is reduced to $92.2 \%$ of its initial value $\left(\varphi_{0 i}\right)$ and for width of $600 \mu \mathrm{m}$, it has reached to $95.2 \%$ of it is initial value. Thus, a lower potential drop $\left(\Delta \varphi_{0}=\varphi_{0 i}-\varphi_{0 f}\right)$ occurs at greater widths, and the probability of re-strike is therefore higher. Consequently, in Fig. 11c, d, the charge $0.0694 \mathrm{mC} / \mathrm{m}^{2}$ accumulated in the $300-\mu \mathrm{m}$ wide micro-cavity is higher than that in the $600-\mu \mathrm{m}$ wide micro-cavity $\left(0.0432 \mathrm{mC} / \mathrm{m}^{2}\right)$. These findings are in agreement with experimental results reported by Auckland et al. [47-49]. He studied the influence of diameter on the quantity of charge accumulating on the side-walls of a micro-cavity in specimens with (a)

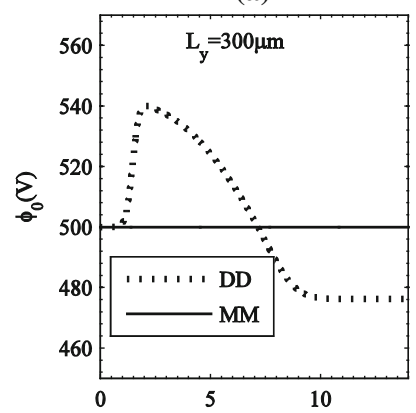

(c)

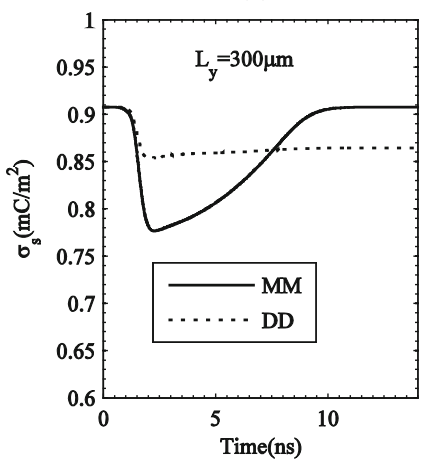

(b)

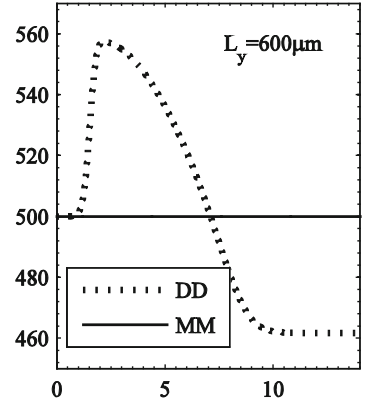

(d)

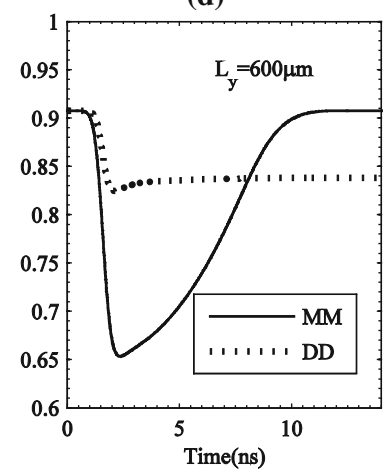

Fig. 11 Time history of the electric potential on the anode dielectric for a width of $L_{\mathrm{y}}=300 \mu \mathrm{m}$, b width of $L_{\mathrm{y}}=600 \mu \mathrm{m}$, and the charge density on the anode dielectric for $\mathbf{c}$ width of $L_{\mathrm{y}}=300 \mu \mathrm{m}$, and d width of $L_{\mathrm{y}}=600 \mu \mathrm{m}$ 
diameters in the range $50-126 \mu \mathrm{m}$ and length of $10 \mathrm{~mm}$. While the model of discharge propagation in their experiments is different (streamer as opposed to pulses), he observed a reduction in the amount of accumulated charges with increasing in diameter of micro-cavity.

These results corroborate the experimental results obtained by Auckland et al. [48], indicating that the electrical strength of gas confined in narrow micro-cavities increases with the length of the micro-cavity and decreases with micro-cavity diameter.

Morshuis et al. [7] reports that tests on voids with small diameters (1-4 mm) show discharge mechanisms similar to larger widths. These findings also show that the effect of width on discharge is negligible.

\section{Gas pressure inside the micro-cavity}

The effect of gas pressure on the nature of PD current pulse is studied in this section. The gas pressure varies from 76 to 1260 Torr, while other parameters are kept constant (Table 1).

The discharge process occurring in cavities, depending upon gas pressure, may assume either a spark, glow or pseudo-glow character. As Morshuis [36] pointed out, in small cavities, the gas plays an important role in the ionization process. The effective ionization coefficient is a (a)

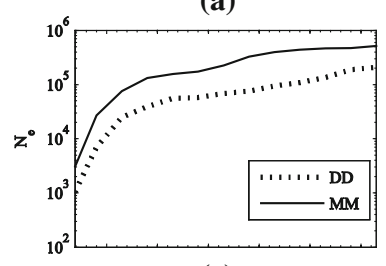

(c)

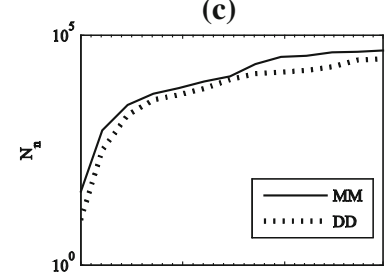

(e)

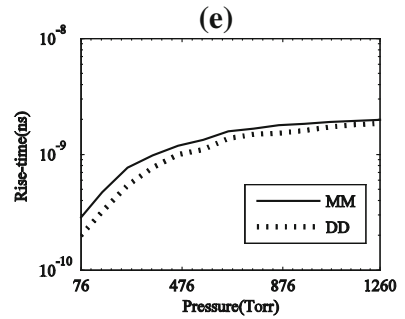

(b)

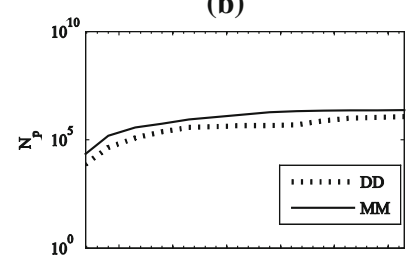

(d)
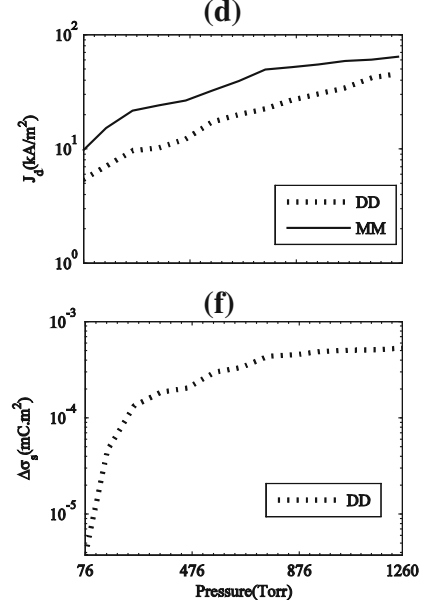

Fig. 12 Variation with gas pressure inside the micro-cavity a number of electrons, $\mathbf{b}$ number of positive ions, $\mathbf{c}$ number of negative ions, d PD current, e discharge current rise-time for both MM and DD configurations, and $\mathbf{f}$ charge accumulated on the dielectrics property of the gas and is therefore affected by the changing gas chemistry and by a change of pressure.

It is seen in Fig. 12a-c that the maximum number of charged particles residing inside the micro-cavity increases with gas pressure. Therefore in Fig. 12d, the magnitude of PD pulse increases. The rise-time of the PD pulse versus gas pressure is presented in Fig. 12e. The effect of gas pressure on the rise-time is significantly high. At lower gas pressures, e.g., 76 Torr, the resultant PD pulse current amplitudes are too low but the rise-time is too rapid and it might be possible to detect them by conventional PD detectors. Gaxiola et al. has reported that with increasing pressure, the time-to-breakdown rapidly increases over two orders of magnitude [30].

However, the experimental findings by Kasten et al. for a cavity with length of $20 \mathrm{~mm}$ show that the time to peak of discharge current decreases as pressure increases [50]. His findings relate to cavities in the millimeter range, where streamers may be taken to control discharge propagation and transfer the results correspond to a different physical mechanism.

The variation of charge accumulated on the anode and cathode dielectrics $\left(\Delta \sigma_{\mathrm{s}}=\sigma_{\mathrm{si}}-\sigma_{\mathrm{sf}}\right)$ versus gas pressure inside the micro-cavity is depicted in Fig. 12f. Owing to higher ionization at higher pressures, larger number of charges (mostly electrons) will reach the dielectric at higher pressures.

As is clear in Fig. 13, for a gas pressure of 260 Torr, the maximum values of PD pulse current is around $416 \mu \mathrm{A}$ for $\mathrm{MM}$ configuration and $256 \mu \mathrm{A}$ for $\mathrm{DD}$ configuration, respectively, while for a gas pressure of 860 Torr, the corresponding values are 520 and $288 \mu \mathrm{A}$, respectively. It may be noted that lower pressure values lead not only to reduced peak values of the PD pulse current, but also cause shorter delays in the formation as well as reduction in the width of PD pulse.

In summary, at higher gas pressures, greater production of charged particles within micro-cavity over time and consequently increase in discharge current as well as charge accumulated on the anode and cathode dielectrics occurs.

\section{Dielectric permittivity}

In homogenous insulating materials, the electric field is distributed uniformly within the entire volume. In nonhomogenous insulating materials, due to manufacturing defects, aging effects, etc., the distribution of the electric field is no longer uniform.

The electric field distributes across two layers of dielectric connected in series in the ratio of their permittivities and thickness (Fig. 2a). In this section, the effects of dielectric permittivity on the behavior of PD pulse are 
(a)

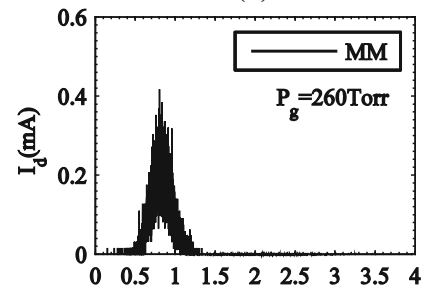

(c)

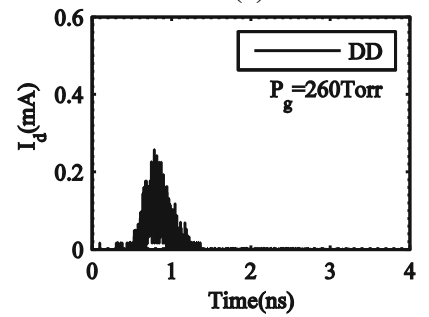

(b)

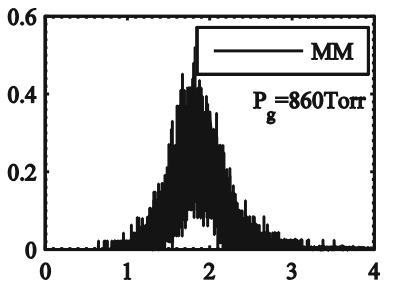

(d)

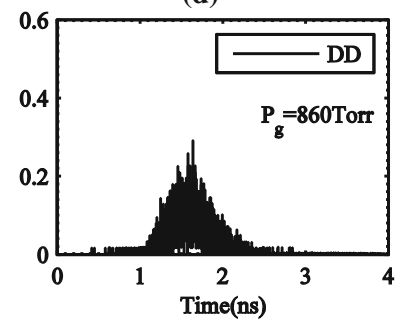

Fig. 13 Time history of PD pulse current for a MM configuration for gas pressure of $P_{\mathrm{g}}=260$ Torr, $\mathbf{b} \mathrm{MM}$ configuration for gas pressure of $P_{\mathrm{g}}=860$ Torr, c DD configuration for gas pressure of $P_{\mathrm{g}}=260-$ Torr, and $\mathbf{d}$ DD configuration for gas pressure of $P_{\mathrm{g}}=860$ Torr

(a)

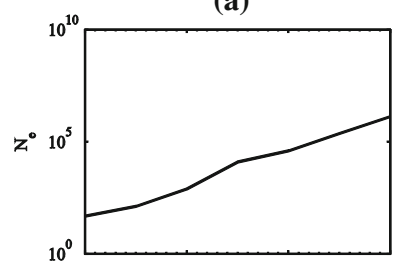

(c)

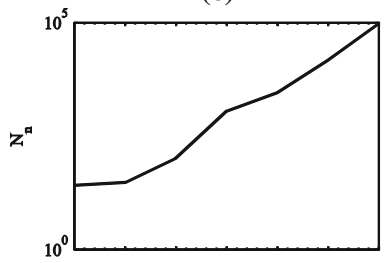

(e)

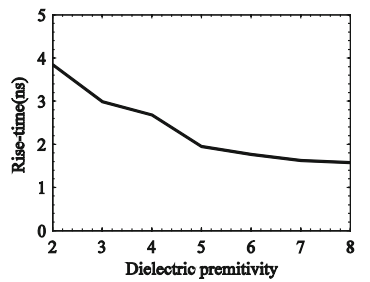

(b)

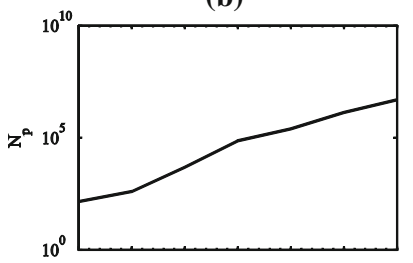

(d)

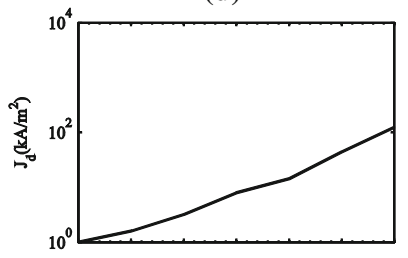

(f)

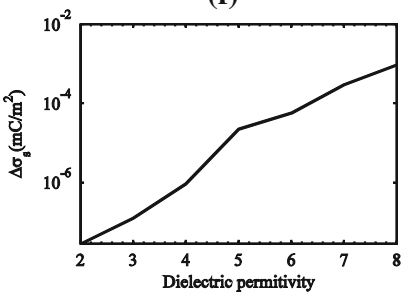

Fig. 14 Variation with dielectric permittivity of a peak value of number of electrons, $\mathbf{b}$ peak value of number of positive ions, $\mathbf{c}$ peak value of number of negative ions, $\mathbf{d}$ PD current density, e PD pulse rise-time, and $\mathbf{f}$ charge accumulated on the dielectrics

studied. The applied electric potential between the metallic electrodes has been kept constant (e.g., $E_{\mathrm{a}}=20 \mathrm{kV} / \mathrm{mm}$ ) for the DD configuration, i.e., the electric potential across the dielectric-gap combination. The permittivity of surrounding material is varied, while keeping the dimensions

and geometry constant. The applied electric field inside the micro-cavity $\left(E_{\mathrm{a}}\right)$ increases and ionization inside the microcavity becomes stronger. Owing to this, as is clear from Fig. 14a-c, the maximum number of charged particles produced inside the micro-cavity increases. The magnitude of the PD pulse as a function of dielectric permittivity is presented in Fig. 14d. Figure 14e shows that the rise-time of the PD pulse falls with $\varepsilon_{\mathrm{r}}$, probably due to increased space charge effects at the dielectric cathode, and decreases with dielectric permittivity as effective electric field along micro-cavity increases.

The variation of charge accumulated on the dielectric anode and cathode $\left(\Delta \sigma_{\mathrm{s}}=\sigma_{\mathrm{si}}-\sigma_{\mathrm{sf}}\right)$ with dielectric permittivity is depicted in Fig. 14f. Owing to increase in the strength of ionization with permittivity of dielectric, the amount of charge accumulated on the dielectrics surface increases.

A point to be noted is that the effect of dielectric permittivity on charge accumulation on the dielectric anode and cathode, PD pulse magnitude, and its rise-time is significant. All simulations reported in the previous sections have been performed with a dielectric permittivity of $\varepsilon_{\mathrm{r}}=5$.

\section{Discussions}

An important contribution of this work is its ability to investigate of the effects of different parameters on PDs inside the micro-cavities for two different dielectric configurations (MM and DD). The calculated PD pulse forms are very much affected by values of micro-cavity length, gas pressure, and dielectric permittivity, while the effect of variations in micro-cavity width is relatively less. These results show that for a kept fix each one of the above parameters, the detected PD pulse form, amplitude and rise-time can exhibit considerable variation due to possible changes in each every above-mentioned parameter. Since the response of a conventional PD detector is a function of the rise-time and pulse amplitude of the PD pulse, variations in the detected pulse would result.

The accuracy of peak pulse measurement would thus be affected in that PD pulse detector would respond more favorably to pulses having rapid rise-times. Consequently, it is quite conceivable that a lower amplitude discharge pulse with a more rapid rise-time may produce a larger indication on that detector than a higher amplitude pulse with a slower rise-time. It would be difficult to resolve this measurement inaccuracy in practice.

By increasing some parameters such as micro-cavity length, gas pressure the space charge density accumulated at the cathode increases and leads to less rapid breakdowns while by increase micro-cavity width and dielectric 
permittivity charge accumulation on the cathode leads to more rapid breakdowns and results in pulses with correspondingly reduced rise-time and augmented magnitudes.

\section{Conclusions}

A two-dimensional kinetic model based on PIC-MCC has been developed to a parametric analysis of nature of PD pulse within micro-cavities. Indeed, this parametric analysis focused especially on micro-cavity dimensions, gas pressure inside the micro-cavity, and dielectric permittivity. It proves the ability of the model in reproducing what is known about the dependence of the nature of PD pulse on the various physical parameters. All the parameters that characterize the proposed model have physical meaning and they can be measured resorting analytical to analytical techniques.

So, determining the dependency of the nature of highly localized intense spark-type PDs to different parameters governing them gives an idea of the physical processes involved in their operations within the micro-cavities. It is expected that the employed model will be a useful tool in designing the solid insulating materials.

In summary, while the peak magnitude of PD pulse current increases with micro-cavity length, gas pressure, and dielectric permittivity, it decreases with increase in width. The rise-time of the PD pulse increases with microcavity length and pressure, but it decreases with microcavity width and dielectric permittivity.

Acknowledgments I would like to acknowledge the Institute of Science, High Technology \& Environmental Sciences for financial support within the Project 1.140-90/2/24.

Open Access This article is distributed under the terms of the Creative Commons Attribution 4.0 International License (http://crea tivecommons.org/licenses/by/4.0/), which permits unrestricted use, distribution, and reproduction in any medium, provided you give appropriate credit to the original author(s) and the source, provide a link to the Creative Commons license, and indicate if changes were made.

\section{Appendix}

The total charge density $\sigma_{\mathrm{T}}$ on the anode metallic electrode may be expressed by:

$$
\sigma_{\mathrm{T}}^{t}=\sum_{j=0}^{N-1} \sigma_{0 j}^{t},
$$

where $\sigma_{0 j}^{t}$ is the surface charge density on anode electrode at time ' $t$ '. The simplified form of Eq. (26) of [29] for a 'simple metallic-metallic' configuration is:
$\sigma_{\mathrm{T}}^{t}=\frac{\varepsilon_{0}}{\Delta x} N \varphi_{0}^{t}-\frac{\varepsilon_{0}}{\Delta x} \sum_{j=0}^{N-1} \varphi_{1 j}^{t}-\frac{\Delta x}{2} \sum_{j=0}^{N-1} \rho_{0 j}$,

where $\varphi_{0}^{t}, \varphi_{1 j}^{t}$, and $N$ are the potential on the anode at ' $t$ ', potential at $x_{1}(=1 \times \Delta x)$ and number of divisions along $y$ axis. Particle ' $p$ ' contributes to $Q_{\mathrm{ij}}$ through the space charge density $\rho_{\mathrm{ij}}$ (bilinear weighting: [29]). By combining equations (A1) and (A2), the Eq. (27) of [29] is obtained and is simplified for a MM configuration as follows:

$$
\begin{aligned}
\varphi_{0}^{t}= & \left(\sigma_{\mathrm{T}}^{t-1}+\frac{1}{A}\left(C V(t)-Q_{\mathrm{c}}^{t-1}+Q_{\text {Conv }}^{t}\right)\right. \\
& \left.+\frac{\Delta x}{2} \sum_{j=0}^{j=N-1} \rho_{0 j}+\frac{\varepsilon_{0}}{\Delta x} \sum_{j=0}^{j=N-1} \varphi_{1 j}^{t}\right)\left(\frac{\varepsilon_{0}}{\Delta x} N+C / A\right)^{-1} .
\end{aligned}
$$

Note that this is Eq. (5) in the paper and is used for the MM case.

The equivalent circuit for DD configuration is shown in Fig. 3b. $C_{\mathrm{g}}$ and $C_{\mathrm{d}}$ represent the equivalent capacitances of the gap and the dielectric layers, respectively, and $J_{\text {Conv }}$ is the discharge current density. The time variation of $\sigma_{\mathrm{T}}$, the total charge density on the driven dielectric electrode, may be obtained from the Kirchhoff's Current Law:

$A \frac{\mathrm{d} \sigma_{\mathrm{T}}}{\mathrm{d} t}=I_{\mathrm{ext}}+A J_{\text {conv }}$,

where $I_{\text {ext }}$ and $\sigma_{\mathrm{T}}$ are the total external current and charge on the dielectric surfaces.

The time variation of voltage drop $\left(U_{\mathrm{d}}\right)$ across the dielectric slab is obtained as:

$C_{\mathrm{d}} \frac{\mathrm{d} U_{\mathrm{d}}}{\mathrm{d} t}=I_{\mathrm{ext}}$.

Figure $3 \mathrm{~b}$ shows a simple external circuit consisting of a voltage source in series with a capacitor coupled to the left electrode. The voltage drop $V_{\mathrm{c}}$ across the capacitor in the external circuit is obtained from voltage balance as,

$V_{\mathrm{c}}=V(t)-2 U_{\mathrm{d}}-\varphi_{0}$

$V(t)$ is the applied voltage source. The discrete finite differenced form of Eq. (A4) can be expressed as:

$A\left(\sigma_{\mathrm{T}}^{t}-\sigma_{\mathrm{T}}^{t-1}\right)=Q_{\mathrm{c}}^{t}-Q_{\mathrm{c}}^{t-1}+Q_{\mathrm{conv}}^{t}$,

where $\int I \mathrm{~d} t=Q_{\mathrm{c}}=C V_{\mathrm{c}}$ is the charge on the capacitor and $Q_{\text {conv }}^{t}$ is the charge deposited on the electrode from the discharge in the cavity during the time interval $(t-1, \mathrm{t})$. Combining Eqs. (A6) and (A7), we obtain:

$\sigma_{\mathrm{T}}^{t}=\sigma_{\mathrm{T}}^{t-1}+\frac{1}{A}\left(C V(t)-C \varphi_{0}^{t}-2 C U_{\mathrm{d}}^{t}-Q_{\mathrm{c}}^{t-1}+Q_{\mathrm{conv}}^{t}\right)$. 
Similarly, the discrete finite differenced form of Eq. (A5) can be expressed as:

$U_{\mathrm{d}}^{t}-U_{\mathrm{d}}^{t-1}=\frac{1}{C_{\mathrm{d}}} \int I \mathrm{~d} t=\frac{Q_{\mathrm{c}}^{t}-Q_{\mathrm{c}}^{t-1}}{C_{\mathrm{d}}}$.

If we combine Eqs. (A6) and (A9), then we have:

$U_{\mathrm{d}}^{t}=\frac{U_{\mathrm{d}}^{t-1}+\frac{1}{C_{\mathrm{d}}}\left(C V(t)-C \varphi_{0}^{t}-Q_{\mathrm{c}}^{t-1}\right)}{1+C / C_{\mathrm{d}}}$.

By inserting the value of $U_{\mathrm{d}}^{t}$ to the Eq. (A8), the value of $\sigma_{\mathrm{T}}^{t}$ can be written as follow:

$\sigma_{\mathrm{T}}^{t}=\sigma_{\mathrm{T}}^{t-1}+\frac{1}{A}\left(\alpha C V(t)-\alpha C \varphi_{0}^{t}-2 \alpha C U_{\mathrm{d}}^{t-1}-\alpha Q_{\mathrm{c}}^{t-1}+Q_{\text {conv }}^{t}\right)$

Equations (A1), (A2) for $\sigma_{\mathrm{T}}^{t}$ and (A11) can be combined and solved for $\varphi_{0}^{t}$ on dielectric anode to produce:

$$
\begin{aligned}
\varphi_{0}^{t}= & \left(\sigma_{\mathrm{s}_{\mathrm{T}}}^{t-1}+\frac{1}{A}\left(\alpha C V(t)-\alpha Q_{\mathrm{c}}^{t-1}-2 \alpha C U_{\mathrm{d}}^{t-1}+Q_{\mathrm{Conv}}^{t}\right)\right. \\
& \left.+\frac{\Delta x}{2} \sum_{j=0}^{j=N} \rho_{0 j}+\frac{\varepsilon}{\Delta x} \sum_{j=0}^{j=N} \varphi_{1 j}^{t}\right) \cdot\left(\frac{\varepsilon}{\Delta x} N+\alpha C / A\right)^{-1} .
\end{aligned}
$$

\section{References}

1. Bartnikas, R., Novak, J.P.: On the character of different forms of partial discharge and their related terminologies. IEEE Trans. Electr. Insul. 28(6), 956-968 (1993)

2. Bartnikas, R., Novak, J.P.: Effect of over-voltage on the rise-time and amplitude of PD pulses. IEEE Trans. Dielectr. Electr. Insul. 2(4), 557-566 (1995)

3. Bartnikas, R.: Partial discharges: their mechanism, detection and measurement. IEEE Trans. Dielectr. Electr. Insul. 9(5), 763-808 (2002)

4. Bartnikas, R.: A comment governing the rise-times of partial discharge pulses. IEEE Trans. Dielectr. Electr. Insul. 12(2), 196-202 (2005)

5. Burgener, H. P., Teich, T. H. Frohljch, K. (2002), Simulations of partial discharges of small micro-cracks parallel to the electrical field in polymeric materials, Annual report CEIDP, pp. 146-150

6. Gu, W.Y., Laurent, C., Mayoux, C.: Characteristics of discharges inside simulated tree micro-cavities under impulse voltage. J. Phys. D 19, 2197-2207 (1986)

7. Morshuis, P.H.F., Kreuger, F.H.: Transition from streamer to Townsend mechanisms in dielectric voids. J. Phys. D 23, 1562-1568 (1990)

8. Hudon, C., Bartnikas, R., Wertheimer, M.R.: Effect of physicochemical degradation of epoxy resin on partial discharge behavior. IEEE Trans. Dielectr. Electr. Insul. 2(6), 1083-1094 (1995)

9. Hudon, C., Bartnikas, R., Wertheimer, M.R.: Spark-to-glow discharge transition due to increased surface conductivity on epoxy resin specimens. IEEE Trans. Dielectr. Electr. Insul. 28(1), 1-8 (1993)

10. Hudon, C., Bartnikas, R., Wertheimer, M. R.: Surface conductivity of epoxy specimens subjected to partial discharges. IEEE
International Symposium on Electrical Insulation, Toronto, Canada, pp. 3-6 (1990)

11. Hudon, C., Bartnikas, R., Wertheimer, M. R.: Chemical and physical degradation effects on epoxy surfaces exposed to partial discharges. 4th International Conference on Properties and Applications of Dielectric Materials, Brisbane Australia, pp. 811-814 (1994)

12. Birdsall, C.K., Langdon, A.B.: Plasma Physics via Computer Simulation. McGraw-Hill, New York (1985)

13. Lawson, W.: Particle simulation of bounded 1D plasma systems. J. Comput. Phys. 80, 253-276 (1989)

14. Lawson, W.: Artificial cooling due to quiet injection in bounded plasma particle simulations. J. Comput. Phys. 77, 330-339 (1988)

15. Hockney, R.W., Eastwood, J.W.: Computer Simulation Using Particles. Taylor \& Francis Group, New York (1988)

16. Radmilovic-Radjenovic, M., Radjenovic, B.: A particle-in-cell simulation of the breakdown mechanism in micro-discharges with an improved secondary emission model. Contrib. Plasma Phys. 47(3), 165-172 (2007)

17. Radmilovic-Radjenovic, M., Lee, J.K., Iza, F., Park, G.Y.: Particle-in-cell simulation of gas breakdown in micro-gaps. J. Phys. D 38(6), 950-954 (2005)

18. Ganjovi, A., Gupta, N., Raju, G.R.G.: A kinetic model of a PD pulse within voids of sub-millimeter dimensions. IEEE Trans. Dielectr. Electr. Insul. 16(6), 1743-1754 (2009)

19. Ganjovi, A., Rastpour, G.: Modeling of distribution of PD pulses within micro-cavities. Int. Rev. Model. Simul. 4(2), 219-227 (2011)

20. Ganjovi, A.: Estimation of photo-degradation of dielectrics surrounding the narrow channel due to PD activity. J. Theor. Appl. Phys. (2014). doi:10.1007/s40094-014-0144-6

21. Birdsall, C.K.: Particle-in-cell charged-particle simulations, plus Monte Carlo collisions with neutral atoms, PIC-MCC. IEEE Trans. Plasma Sci. 19, 65-85 (1991)

22. Vahedi, V., Surendra, M.: Monte-Carlo collision model for particle-in-cell method: application to argon and oxygen discharges. Comput. Phys. Commun. 87, 179-198 (1995)

23. Raju, G.R.G.: Gaseous Electronics: Theory and Practice. Taylor and Francis, Boca Raton (2005)

24. Roldan, A., Perez, J.M., Williart, A., Garcia, B.: Energy deposition model for low-energy electrons $(10-10000 \mathrm{eV})$ in air. J. Appl. Phys. 95(10), 5865-5870 (2004)

25. Zhang, W., Fisher, T.S., Garimella, S.V.: Simulation of ion generation and breakdown in atmospheric air. J. Appl. Phys. 96(11), 6066-6072 (2004)

26. Mardahl, P.J., Cartwright, K.L.: "Reducing Numerical Heating in 1-D PIC Simulations”, Annual Progress Report for 1993, Plasma Theory and Simulation Group. University of California Technical Memorandum, Electronics Research Laboratory, Berkeley (1993)

27. Mardahl, P.J., Verboncoeur, J.P.: Charge conservation in electromagnetic PIC codes; spectral comparison of Boris/DADI and Langdon-Marder methods. Comput. Phys. Commun. 106, 219-229 (1997)

28. Verboncoeur, J.P.: Symmetric spline weighting for charge and current density in particle simulation. J. Comput. Phys. 174, 421-427 (2001)

29. Vahedi, V., DiPeso, G.: Simultaneous potential and circuit solution for two-dimensional bounded plasma simulation codes. J. Comput. Phys. 131, 149-163 (1997)

30. Gaxiola, E. H. R., Wetzer, J. M., Partial discharges in micrometer voids PTFE. IEEE International Symposium on Electrical Insulation, Quebec, Canada, pp. 16-19 (1996)

31. Novak, J. P., Bartnikas, R., Quantitative model of a short-gap breakdown in air. XVIIth International symposium on discharge and electrical insulation in vacuum, Berkeley, U.S., pp. 60-64 (1996) 
32. Bartnikas, R.: Some observations on the character of corona discharges in short gap spaces. IEEE Trans. Electr. Insul. 2(2), 63-75 (1971)

33. Devins, J.C.: The physics of partial discharges in solid dielectrics. IEEE Trans. Electr. Insul. EI-19(5), 475-495 (1984)

34. Mazzanti, G., Montanari, G.C., Civenni, F.: Model of inception and growth of damage from microvoids in polyethylene-based materials for HVDC cables part 1: theoretical approach. IEEE Trans. Dielectr. Electr. Insul. 14(5), 1242-1254 (2007)

35. Mazzanti, G., Montanari, G.C., Civenni, F.: Model of inception and growth of damage from microvoids in polyethylene-based materials for HVDC cables part 2: parametric investigation and data fitting. IEEE Trans. Dielectr. Electr. Insul. 14(5), 1255-1263 (2007)

36. Morshuis, P.: Assessment of dielectric degradation by ultrawideband PD detection. IEEE Trans. Dielectr. Electr. Insul. 2(5), 744-760 (1995)

37. Wetzer, J.M., van der Laan, P.C.T.: Pre-breakdown currents: basic interpretation and time-resolved. IEEE Trans. Electr. Insul. 24(2), 297-308 (2989)

38. Morshuis, P.: Partial discharge mechanisms in voids related to dielectric degradation. IEE Proc. Sci. Meas. Technol. 142(1), 62-68 (1995)

39. Novak, J.P., Bartnikas, R.: Effect of dielectric surfaces on the nature of partial discharges. IEEE Trans. Dielectr. Electr. Insul. 7(1), 146-151 (2000)

40. Morshuis, P.: Partial discharge mechanisms in voids related to dielectric degradation. IEE Proc. Sci. Meas. Technol. 142(1), 62-68 (1995)

41. Serra, S., Montanari, G.C., Mazzanti, G.: Theory of inception mechanism and growth of defect-induced damage in polyethylene cable insulation. J. Appl. Phys. 98, 034102-1-03410203410215 (2005)
42. Ramachandra, B., Nema, R.S.: Characterization of partial discharge pulses in artificial voids in polypropylene films used in capacitors. IEEE International Symposium on Electrical Insulation, Montreal, Canada, pp. 517-520 (1996)

43. Novak, J.P., Bartnikas, R.: Ionization and excitation behavior in a micro-cavity. IEEE Trans. Dielectr. Electr. Insul. 2(5), 724-728 (1995)

44. Nikonov, V.: Master Thesis, Influence of Electrode Surface Charge Accumulation upon Partial Discharge Behavior. University of Montreal, Canada (1999)

45. Wu, K., Suzuoki, Y., Mizutani, T., Xie, H.: Model for partial discharges associated with treeing breakdown: I. PDs in tree channels. J. Phys. D 33, 1197 (2000)

46. Serdyuk, Y.V., Gubanski, S.M.: Computer modeling of interaction of gas discharge plasma with solid dielectric barriers. IEEE Trans. Dielectr. Electr. Insul. 12(4), 725 (2005)

47. Auckland, D. W., Borishade, A. B., Cooper, R.: The breakdown characteristics of air-filled tubules in solid insulation. IEEE Conference Publication, Dielectric Materials, Measurements and Applications, Cambridge, Vol. 129, p. 15 (1975)

48. Auckland, D.W., Borishade, A.B., Cooper, R.: Photographic investigation of breakdown of composite insulation. Proc. IEE 124(12), 1263 (1977)

49. Auckland, D.W., Kabir, S.M.F., Varlow, B.R.: Charge deposition in gas filled channels with insulating walls. IEE Proc. A 140(6), 509-516 (1993)

50. Kasten, D.G., Liu, X., Sebo, S.A., Grosjean, D.F., Schweickart, D.L.: Partial discharge measurements in air and argon at low pressures with and without a dielectric barrier. IEEE Trans. Dielectr. Electr. Insul. 12(2), 362-373 (2005) 